\title{
Synthesis and Characterization of Conducting Polymer Nanorods by Using Electropolymerization in Nanoporous Template
}

\author{
Yuko MAEDA, ${ }^{\text {a }}$ Fumiko CHIBA, ${ }^{\mathrm{b}}$ Kikuno IIDA, ${ }^{\mathrm{b}}$ Kenro TOTANI, ${ }^{\mathrm{b}}$ Kenji OGINO, ${ }^{\mathrm{a}}$ \\ Takayuki ISHIBASHI, ${ }^{\mathrm{c}}$ Hari Singh NALWA, ${ }^{\mathrm{d}}$ and Toshiyuki WATANABE ${ }^{\mathrm{b}}$
}

\author{
${ }^{a}$ Graduate School of Bio-Applications Systems and Engineering, Tokyo University of Agriculture and Technology (2- \\ 24-16 Nakacho, Koganei, Tokyo 184-8588, Japan) \\ ${ }^{b}$ Department of Organic and Polymer Materials Chemistry, Faculty of Technology, Tokyo University of Agriculture \\ and Technology (2-24-16 Nakacho, Koganei, Tokyo 184-8588, Japan) \\ 'Department of Applied Physics, Faculty of Technology, Tokyo University of Agriculture and Technology (2-24-16 \\ Nakacho, Koganei, Tokyo 184-8588, Japan) \\ dStanford Scientific Corporation (25650 North Lewis Way, Stevenson Ranch, CA 91381, USA)
}

Received December 1, 2003 ; Accepted March 4, 2004

\begin{abstract}
Uniform size nanorods consisting of polypyrrole were synthesized via electropolymerization using a porous alumina template. The dispersion of polymer particles was obtained by dissolving the template in an alkaline solution followed by dialysis with distilled water. SEM-EDS and TEM techniques revealed that synthesized polypyrrole nanoparticles are rod-shaped having a diameter of $20 \mathrm{~nm}$.
\end{abstract}

Key Words : Conducting Polymer Nanorods, Polypyrrole, Nanoporous Template, Electropolymerization

\section{Introduction}

Recently much attention has been paid for nanoparticles in the size range between isolated molecules and bulk materials due to their unique intermediated state. '. ' If the size of a particle decreases below several tens of a nanometer, then the physical properties of materials drastically change. ${ }^{3,4)}$ These nanoparticles can be used in a variety of applications such as catalyst, ${ }^{5,6}$ ) chemical sensors, ${ }^{7)}$ nanoelectronics, ${ }^{2,3}{ }^{3}$ optical devices $^{2,3)}$ etc. The nanoparticles of semiconductors, ${ }^{8-11)}$ metals, ${ }^{12-14)}$ and ceramics ${ }^{15-18)}$ have been developed.

Polymer nanoparticles are synthesized via emulsion polymerization. ${ }^{19)}$ Nakanishi et al.$^{4,20)}$ proposed a reprecipitation method to fabricate nanocrystals from aqueous dispersion. However, a very little attention has been focused on polymeric nanoparticles having anisotropic shape. Interestingly, the rod-shaped particles dispersed in fluids are sensitive to external fields which may lead to birefringence or optical switching. According to Gans theory, ${ }^{21)}$ the optical properties of rod-shaped metal nanoparticles can be tuned by a geometrical factor, which is an extension of the Mie theory. ${ }^{22}$ The control of the particle size is very important for opto-electronic devices. In order to control the aspect ratio of rod-shaped particles, the electrodeposition in nanoporous alumina template was developed and applied to production of rod-shaped gold ${ }^{23)}$ and $\mathrm{Bi}_{2} \mathrm{Te}_{3}$ particles. Anodic oxidation method offers several advantages that nanopores can be produced via a simple operation, and the pore size could be easily controlled by reaction conditions such as applied voltage, electrolytes, and oxidation temperature. Recently, Martin et $a l{ }^{24,25)}$ and Joo et $a l{ }^{26)}$ synthesized nanotube and nanowires of conducting polymer having diameter of 200 $\mathrm{nm}$ by using nanoporous template through electropolymerization method, however. no polymer rod dispersion was obtained and Chemical composition of these rods were not confirmed at all. In this article, we report a method to prepare dispersion of rod-shaped nanoparticles of polypyrrole having uniform size ( $20 \mathrm{~nm}$ of diameter). The various steps involve anodization of an aluminum substrate, the electropolymerization of polypyrrole followed by dissolution of aluminum oxide, and finally releasing the rods in an aqueous solution. Chemical composition of these rods were confined by SEM-EDS. The synthesized nanorods were isolated to obtain a direct image through the electron microscopy.

\section{Experimental}

Nanorods of polypyrrole (PPy) were synthesized in nanopores of an anodic aluminum oxide by electropolymerization method. Anodic aluminum oxide template was prepared by using a modified procedure reported in literature. ${ }^{27)}$ In a standard procedure, an aluminum substrate (Furuuchi Chemical, 99.99\%, $1 \mathrm{~cm} \times 5 \mathrm{~cm} \times 0.5$ $\mathrm{mm}$ ) was washed with ethanol and acetone, followed by electropolishing step in a mixture of $12.5 \mathrm{~g}$ of chromic anhydride, $200 \mathrm{ml}$ of phosphoric acid, $50 \mathrm{ml}$ of sulfuric acid and $10 \mathrm{ml}$ of distilled water. 10 volts of voltage was applied to anodic aluminum sheet by using a direct current power supply (Toyo Technica Corporation, Source Meter 2400) for electropolishing and the reaction was terminated when the current density was decreased to $0.05 \mathrm{~A} / \mathrm{cm}^{2}$. The reaction temperature was controlled by thermostat in the range from 70 to $80^{\circ} \mathrm{C}$. 
In order to produce a porous alumina plate, anodic oxidation was carried out as follows. Electrolyte was 8.75 $\mathrm{v} / \mathrm{v} \%$ of sulfuric acid. The distance between two electrodes was $1.8 \mathrm{~cm}$, and 15 volts of voltage was applied for $60 \mathrm{~min}$ at $18^{\circ} \mathrm{C}$. After the anodization, the aluminum sheet was sonicated in pure water and washed with 100 $\mathrm{g} / \mathrm{dm}^{3}$ phosphoric acid. In addition to first anodic oxidation, second anodic oxidation was performed for further thinning of the barrier layer. Electrolyte was $100 \mathrm{~g} / \mathrm{dm}^{3}$ of phosphoric acid. The distance between two electrodes was $1.8 \mathrm{~cm}$, and 8 volts of voltage was applied for $5 \mathrm{~min}$ at $18^{\circ} \mathrm{C}$. As shown in Fig. 1, scanning electron microscope image revealed that uniform size of pores $(20 \mathrm{~nm})$ were produced under these conditions. We assumed that thickness of barrier layer were reduced from $14 \mathrm{~nm}$ to 5 $\mathrm{nm}$ by measuring current density of second anodic oxidation.

\section{1 Polymerization}

Acetonitrile dehydrated (Wako), pyrrole (TCI), lithium perchlorate anhydrous (Kishida Chemical) were used without further purification. Acetonitrile solution of pyrrole $\left(0.05 \mathrm{~mol} / \mathrm{dm}^{3}\right)$ and lithium perchlorate anhydrous $\left(0.1 \mathrm{~mol} / \mathrm{dm}^{3}\right)$ was prepared for electropolymerization. Before polymerization, dissolved oxygen was removed from the solution by dry nitrogen bubbling for 25 minutes. The second anodic oxidized aluminum template was used as a working electrode and $\mathrm{Pt}$ sheet $(99.9 \%$ Tanaka Kikinzoku) was used as a counter electrode. The reference electrode was a saturated calomel electrode. Electropolymerization was carried out at ambient temperature at a constant voltage of $1.0 \mathrm{~V}$ vs. SCE using a potentiostat (640 A electro analyzer, BAS).

Seven pieces of electropolymerized aluminum template were dipped in $1.25 \mathrm{~mol} / \mathrm{dm}^{3} \mathrm{NaOH}$ solution to dissolve and sonicated until generation of hydrogen. The solution was acidized by copious amount of $1.25 \mathrm{~mol} / \mathrm{dm}^{3} \mathrm{HCl}$ solution and dialyzed with amount of water until neutralized. The solution was condensed to obtain PPy nano rods dispersed solution without surfactant.

\section{2 Characterization}

Both Template and nanoparticles were characterized by energy dispersive $\mathrm{x}$-ray spectrometer equipped on field-emission scanning electron microscope (FE-SEM-

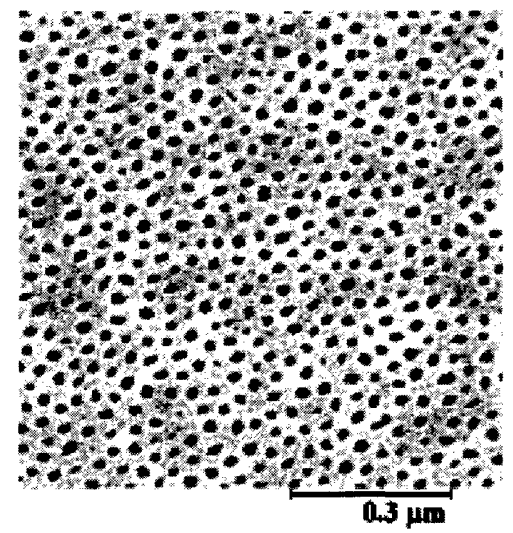

Fig. 1 FE-SEM image of top view of aluminum oxide nanoporous template.
EDS) before and after polymerization (Hitachi S-4500, JEOL JSM-6700 F). Isolated nanorods were observed by transmission electron microscope (TEM) (Hitachi H-700 $\mathrm{H})$. The samples for TEM observation were prepared by dropping the purified aqueous nanorods dispersion on the microgrid membrane (Nisshin EM) followed by drying in air for a week.

\section{Results and Discussion}

After anodization of aluminum, the aqueous solution was gradually changed to monomer solution for electropolymerization. When electropolymerization was immediately performed by using the alumina template with 20 $\mathrm{nm}$ pores in diameter, inhomogeneous distribution of PPy nanorods was obtained as shown in Fig. 2(a) due to slow and inhomogeneous diffusion process of monomer solution in nanopores. In order to improve this problem, porous alumina template was sonicated in the monomer solution for $1 \mathrm{hr}$. after washing the template by water and acetonitrile. This procedure provided improved uniformity of PPy nanorods in the template as shown in Fig. 2(b). No vacant holes were observed in the alumina template. No minor difference of morphology was observed in any places of nano porous template after electropolymerization.

Since PPy is insoluble in solvents and not transparent even in infrared region, it was difficult to confirm the ex-
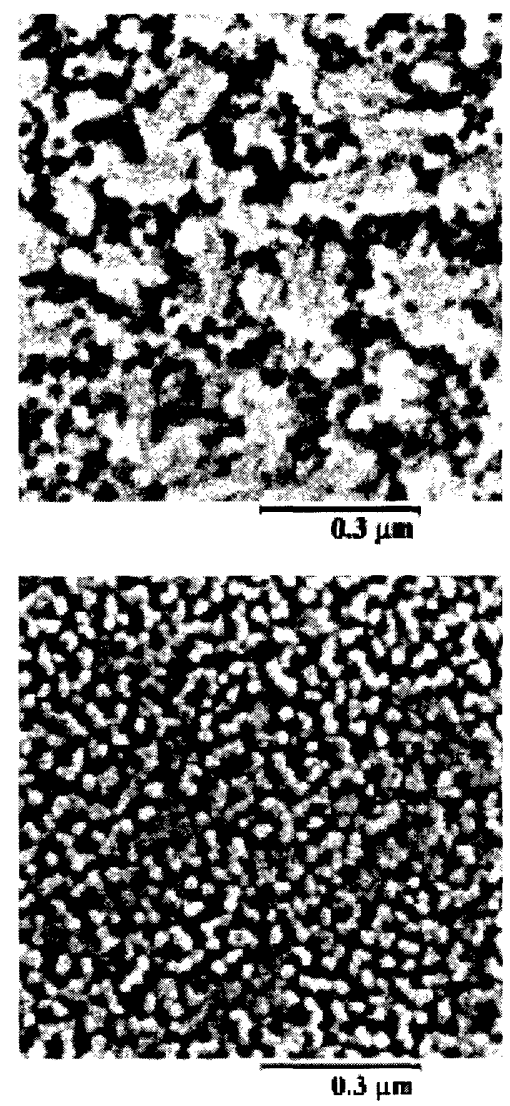

Fig. 2 FE-SEM image of top view of aluminum oxide nanoporous templates after electropolymerization. a) inhomogeneous distribution of PPy nanorods (without ultrasonic treatment) b) homogeneous distribution of PPy nanorods (in the presence of ultrasonic treatment). 
act composition. Therefore, we measured composition of PPy nanorods in the alumina template by using FE-SEMEDS method. As shown in Fig. 3(a), two peaks associated with carbon and nitrogen clearly appeared, whereas only weak peaks of carbon were observed in nanoporous alumina template (control sample) as shown in Fig. 3(b), which support the formation of nanorods of PPy in the alumina template.

Figure 4 shows TEM images of the rod-shaped PPy nanoparticles. To the best of our knowledge, this image is the first direct observation of nanorods in an isolated state. During the dry process, each PPy rods aggregate and then form twisted nanobundles of rods as shown in Fig. 4. Martin et al. ${ }^{24}$ observed PPy tubules since PPy preferentially nucleates and grows on the pore walls. In
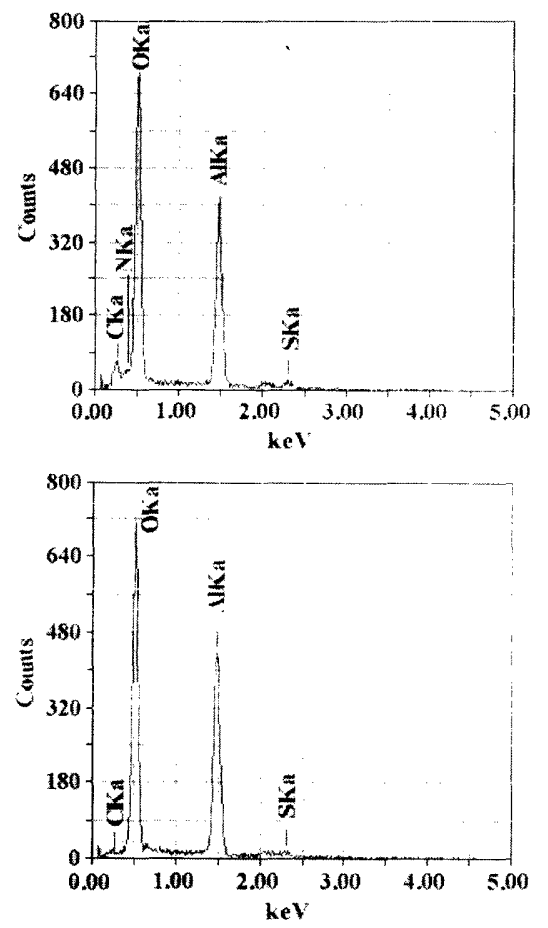

Fig. 3 EDS of alumina template. a)nanoporous alumina template filled by electropolymerized PPy nanorods b) nanoporous alumina template (control sample).

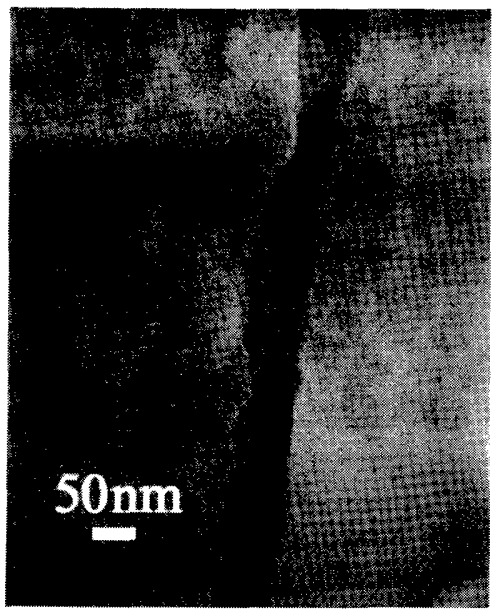

Fig. 4 Transmission electron microscope image of isolated nanobundles. The bundle consists of four nanorods. contrast with Martin's results only PPy rods were obtained in this experiment due to narrow pore of aluminum template. The typical diameter of nanoparticles is 20 $\mathrm{nm}$, which agree well with the diameter of nanopores as shown in Fig. 1. Length of nanoparticles is tuned between $50 \mathrm{~nm}$ and $10 \mu \mathrm{m}$ by polymerization time. Figure 5 shows TEM image of aggregated nano rods through low magnification. The length of aggregated nano rods $\mathrm{L}$ are about 55\% compared with thickness of aluminum template in Fig. 5. The standard deviation of rod length $\sigma_{\mathrm{x}}$ is about $1.1 \mu \mathrm{m} . \sigma_{\mathrm{x}} / \mathrm{L}$ is about $10 \%$ which shows good agreement with Zande's ${ }^{23}$ results for gold rods. Although Joo et al. ${ }^{24)}$ fabricated conducting polymer nanotubes by using camphorsulfonic acid (CSA) dopant, only nanorods were obtained in our experiments. It is not clear whether such results are attributed to dopants, different diameter of nanopores, time of polymerization or current density. In our process, the polymerization was fully accomplished within 15 seconds due to thin barrier layer, whereas other process was performed over a period of 15-40 munutes. In our experiment, the low current density of $1.66 \times 10^{-5} \mathrm{~A} / \mathrm{cm}^{2}$ and thin barrier layer may have improved the uniformity of nanoparticles.

\section{Conclusions}

We have synthesized rod-shaped polypyrrole dispersions through the electropolymerization of pyrrole followed by dissolution of aluminum oxide, and releasing of the rods in an aqueous solution. Thin barrier layer and ultrasonic treatment play important role for uniform polymerization. The diameter of rod was precisely controlled by the pore size of template. The chemical composition of rod-shaped polypyrrole was confirmed by FESEM-EDS method. Currently, we are studying the effect of aspect ratio on electrical and optical properties of conducting nanorods that will be reported elsewhere.

\section{Acknowledgements}

This work was supported in part by the Ministry of

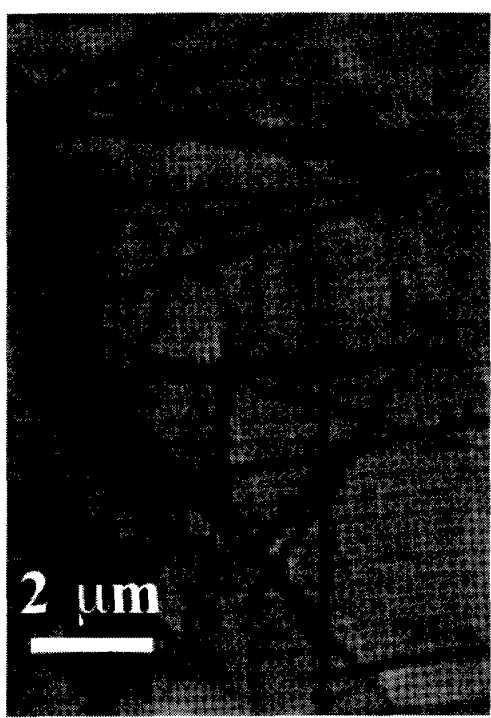

Fig. 5 Transmission electron microscope image of aggregated PPy nano rods. 
Education, Science, Sports and Culture, Japan, in order to fulfill the Grant-in-Aid for Scientific Research on Priority Areas (Molecular synchronization), 11167220 , 2001-2003, and Grant-in-Aid for Exploratory Research (B), 15651069, 2002-2003 and the 21st Century COE (Center of Excellence) program on "Future Nano-Materials" (conducted at Tokyo University of Agriculture \& Technology).

\section{References}

1) M.-I. Baraton, Ed., Synthesis, Functionalization and Surface Treatment of Nanoparticles, American Scientific Publishers, Los Angeles (2003).

2) C. R. Martin, Science, 266, 1961 (1994).

3) Y. Zhang, X. Wang, M. Ma, D. Fu, N. Gu, Z. Lu, J. Xu, L. $\mathrm{Xu}$, and K. Chen, J. Colloid Interface Sci., 266, 377 (2003).

4) H. Nakanishi and H. Takagi, Supermol. Sci., 5, 289 (1998).

5) M. P. Andrews and G. A. Ozin, J. Phys. Chem., 90, 2929 (1986).

6) Y. Nakao and K. Kaeriyama, J. Colloid Interface Sci., 131, 186 (1989).

7) C. A. Mirikin, R. L. Letsinger, R. C. Mucic, and J. J. Storhoff, Nature, 382, 607 (1996).

8) H. Weller, Angew. Chem. Int. Ed. Engl., 32, 41 (1993).

9) A. Henglein, Pure. Appl. Phys., 56, 1215 (1984).

10) R. Rossetti, J. L. Ellison, J. M Gibson, and L. Brus, J. Chem. Phys., 80, 4464 (1984).

11) R. Rossetti, R. Hull, and J. M. Gibson, J. Chem. Phys., 82, 552 (1985).
12) M. Brust, J. Fink, D. Bethel, D. J. Schriffren, and K. F. Jensen, J. Chem. Soc. Chem. Commun., 1995, 1655.

13) J. Tanori and M. P. Pileni, Adv. Mater., 7, 862 (1995).

14) C. B. Murray, C. R. Kagan, and M. G. Bawendi, Annu. Rev. Mater. Sci, , 30, 545 (2000).

15) W. R. Cannon, S. C. Danforth, J. S. Haggerty, and R. A. Marra, J. Am. Ceram. Soc., 65(7), 324 (1982).

16) G. R. Kargedov and N. Z. Lyakhov, Nanostruct. Mater., 11, 559 (1999).

17) D. Ravichandran, R. Roy, P. Ravindranathan, and W. B. White, J. Am. Ceram. Soc., 82, 1082 (1999).

18) G. Skandan, Y. J. Chen, N. Glumac, and B. H. Kear, Nanostruct. Mater., 11, 149 (1999).

19) J. A. Alduncin, J. Forcada, and J. M. Asua, Macromolecules, 27, 2256 (1994).

20) Y. Komai, H. Kasai, and H. Nakanishi, Jpn. J. Appl. Phys., 38, L 81 (1999).

21) R. Gans, Ann. Phys., 37, 881 (1912).

22) G. Mie, Ann. Phys., 25, 377 (1908).

23) B. M. I. van der Zande, M. R. Böhmer, L. G. J. Fokkink, and C. Schönenberger, Langmuir, 16, 451 (2000).

24) C. R. Martin, Sience, 266, 1961 (1994).

25) L. S. Van Dyke and C. R. Martin, Langmuir, 6, 1118 (1990).

26) J. Joo, K. T. Park, B. H. Kim, M. S. Kim, S. Y. Lee, C. K. Jeong, J. K. Lee, D. H. Park, W. K. Yi, S. H. Lee, and K. S. Ryu, Synth. Met., 135, 7 (2003).

27) H. Masuda, K. Yada, and A. Osaka, Jpn. J. Appl. Phys., 37, 1340 (1998). 